\title{
Concerning the Lower Atmosphere Responses to Magnetospheric Storms and Substorms
}

\author{
P. A. Sedykh and I. Yu. Lobycheva \\ Institute of Solar-Terrestrial Physics SB RAS, Lermontov Street, 126 a, P.O. Box 291, Irkutsk 664033, Russia \\ Correspondence should be addressed to P. A. Sedykh; pvlsd@iszf.irk.ru
}

Received 31 January 2013; Revised 22 April 2013; Accepted 25 April 2013

Academic Editor: Helena A. Flocas

Copyright ( 2013 P. A. Sedykh and I. Yu. Lobycheva. This is an open access article distributed under the Creative Commons Attribution License, which permits unrestricted use, distribution, and reproduction in any medium, provided the original work is properly cited.

\begin{abstract}
The issue of existence and physical mechanism for solar-terrestrial couplings has rather a long history. Investigations into the solar activity effect on meteorological processes in the lower atmosphere have become especially topical recently. The aim of this study is to investigate the effect of geomagnetic activity on meteorological processes in the atmosphere. We analyze the data on magnetic storms and tropical cyclones that were observed in the North Atlantic, East Pacific, and West Pacific to understand the mechanism for magnetospheric disturbance effects on complicated nonlinear system of atmospheric processes.
\end{abstract}

\section{Introduction}

There exist statistical correlations among geomagnetic activity, atmospheric pressure, and temperature [1-3]. Authors in [4] suggested that the observed climate response to solar variability is caused by a dynamical response in the troposphere to heating predominantly in the stratosphere.

According to [5], a tropical cyclogenesis may be "a mechanism for effective discharge of the surplus heat in the atmosphere under the conditions when the routine mechanism effect becomes insufficient." Between the solar-terrestrial disturbance parameters, on the one hand, and the cyclogenesis characteristics, on the other, various researchers endeavor to trace hard-to-detect statistical associations. In [6], the correlation between tropical cyclones and the Cycle- 23 storms was investigated. The revealed coincidence between the time of origin and evolution of the 2005 August 23-24 Hurricane Katrina with the powerful geomagnetic storm main phase [7] also boosted the research in this area.

The issue of physical mechanism for solar-terrestrial couplings has long interested researchers. Many geophysicists were almost prepared to reject the idea about a solar activity effect on the lower atmosphere condition as absolutely unacceptable. And, first of all, the matter was that the atmospheric process power enormously exceeds the solar-wind input energy flux into the near-Earth space. Due to this, it seems most unlikely that solar activity could significantly affect the lower atmosphere condition. However, the research done over the last years allowed us to find a clue to overcome this inconsistency. The main objection to a possibility of the solar activity effective influence on the lower atmosphere condition and on weather, based on insufficient power of the solar wind, appears quite surmountable; see, for example, [8]. Also, like the computations in [9] show, the energy necessary to create the atmospheric optical screen (shield) is incomparably lower than the variation amplitude in the screen-induced solar energy flux arriving at the lower atmosphere.

According to [8], variations in the atmospheric ionizing radiation flux observed during geomagnetospheric disturbances cause a noticeable variation in the chemical composition and contents of small components, as well as in the atmosphere transparency. The main types of such variations are [8] (1) galactic cosmic ray intensity short-term depressions (observed during geomagnetic disturbances (Forbush decreases)) caused by dispersion of energetic charged particles by the magnetic fields transported from the solar atmosphere by the solar wind high-velocity streams; (2) solar cosmic ray flux bursts caused by solar flares.

This paper aims to investigate geomagnetic activity effects on meteorological processes and a possible effect of magnetospheric disturbances on the tropical cyclogenesis evolution character. Usually, researchers considered magnetospheric 


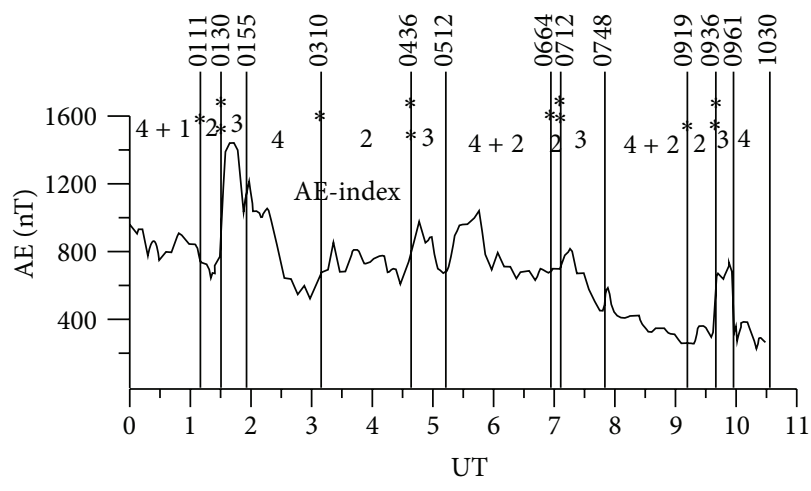

(a)

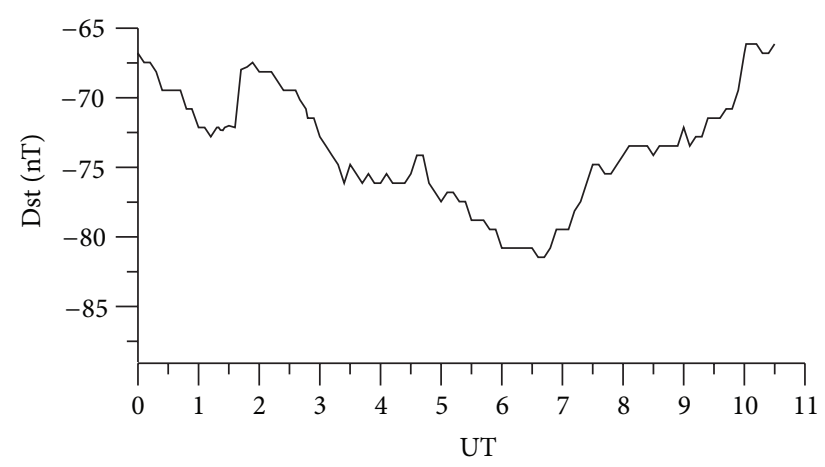

(b)

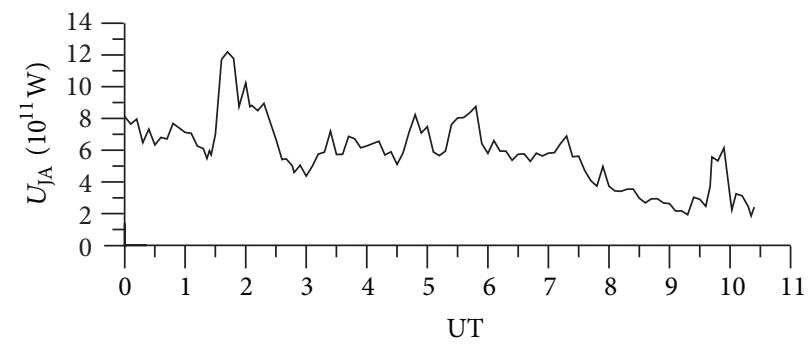

(c)

FIGURE 1: (a) AE-index for the chain of substorms, May 3, 1986. Timing of the chain of substorms CDAW9C [13]. Single and double asterisks indicate the actively convective phase and the expansion (explosive) phase of the substorm, respectively. (b) Dst-index for the chain of CDAW9C substorms [13]. (c) Estimations of power of Joule heating of the ionosphere and power of particles precipitation into the ionosphere for the chain of CDAW9C substorms.

and atmospheric problems in separate papers. This paper is an attempt to combine consistent parts taken from the meteorology ionospheric and magnetospheric physics.

\section{Principal Results}

A magnetospheric storm is a 1-3 day long phenomenon spanning all the magnetosphere regions, and it features sharp depressions in the magnetic field. During storms and substorms, the ionosphere undergoes rather significant Joule heating with a great power of precipitating energetic particles. Huge energy increases the ionosphere temperature and causes large-scale ion drifts and neutral winds.

We used magnetograms from ground-based stations, AEindices, Dst-indices for each magnetospheric storm, and calculated some parameters characterizing energetic aspects of magnetospheric disturbances. The aim was to investigate a possible effect of powerful magnetospheric storms on the evolution character of meteorological processes in the atmosphere to study the correlation between magnetospheric disturbances and meteorological background variations. We also address the meteorological data for 10-day periods after these disturbances.

The power of the ionosphere's Joule heating $U_{j}=$ $\int_{S} \Sigma_{P} E^{2} d S$ is either calculated through the Assimilative Mapping of Ionospheric Electrodynamics (AMIE) [10] or estimated based on empirical formulas as the AE-index function [11]: $U_{j}=0.33 \times \mathrm{AE}$, where $U_{j}$ is in GWatt and the AE-index is in nT. The power of the energetic particles precipitating into the ionosphere $U_{a}$ can be also estimated based on empirical formulas $U_{a}=1.75 \times(\mathrm{AE} / 100+1.6) \times$ $10^{10}(\mathrm{~W})$ [12]. We also used the databases for hurricanes at http://www.nhc.noaa.gov/pastall.shtml, http://www.csc.noaa .gov/hurricane_tracks, and http://www.aoml.noaa.gov/hrd/ hurdat/ushurrlist18512007.txt. To study the surface pressure, the temperature, and other characteristics, we used the data at $\mathrm{ftp}: / / \mathrm{ftp} . \mathrm{cdc}$.noaa.gov/. The information on tropical cyclones was obtained from http://russian.wunderground.com/ hurricane/hurrarchive.asp? region=at.

The 1986 May 3 magnetospheric storm over the (00001100) UT interval was studied within the international CDAW9C (Coordinated Data Analysis Workshop) Project and represented a 4-substorm chain [13]. This event refers to magnetospheric storms that occurred during solar Cycle-21. It featured the AE-index reaching the values of about 1400$1500 \mathrm{nT}$, whereas the Dst-index reached only -70-80 nT (see Figures 1 and 2). Because this storm comprised 4 substorms, we detected the substorm phases by timing each of the 4 substorms. Following [14, 15], we will term these phases the growth phase (1), the actively-convective phase (2), the expansion (explosive) phase (3), and the recovery phase (4).

In summary, the data on the 1986 May 3 event (Figures 1 and 2) could be used to trace each substorm phase contribution (energy loading-unloading) to the composite set of processes in the lower atmosphere. 


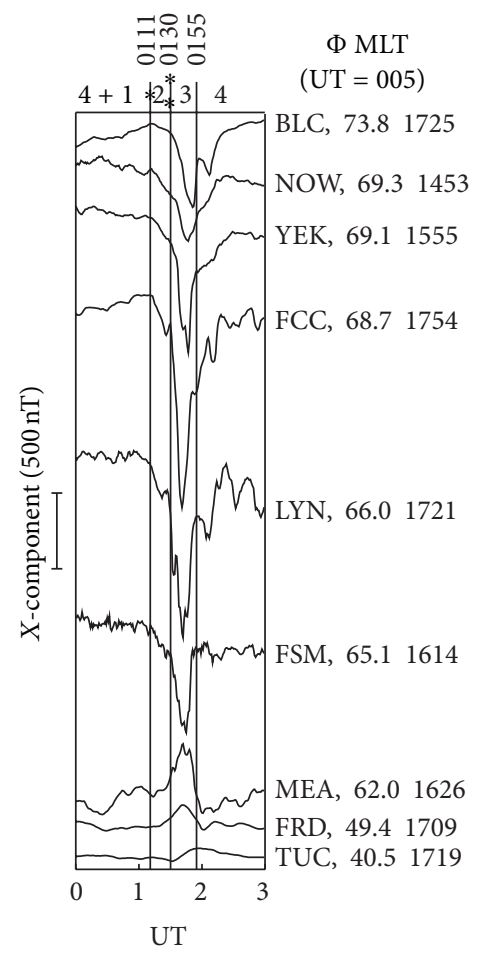

(a)

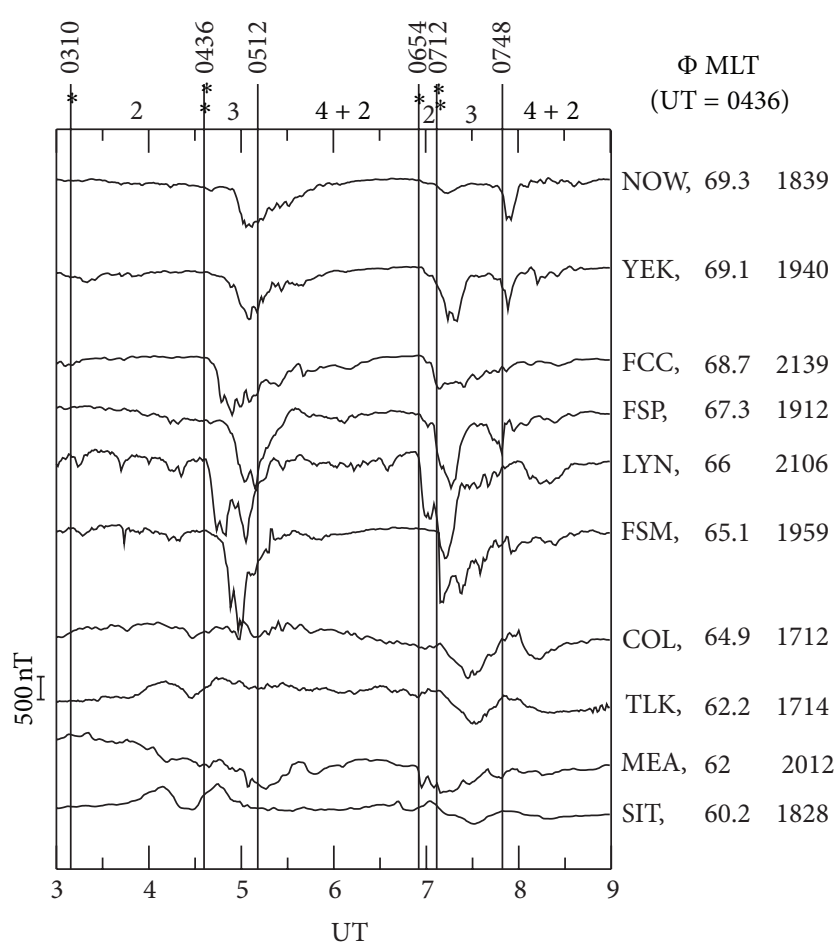

(b)

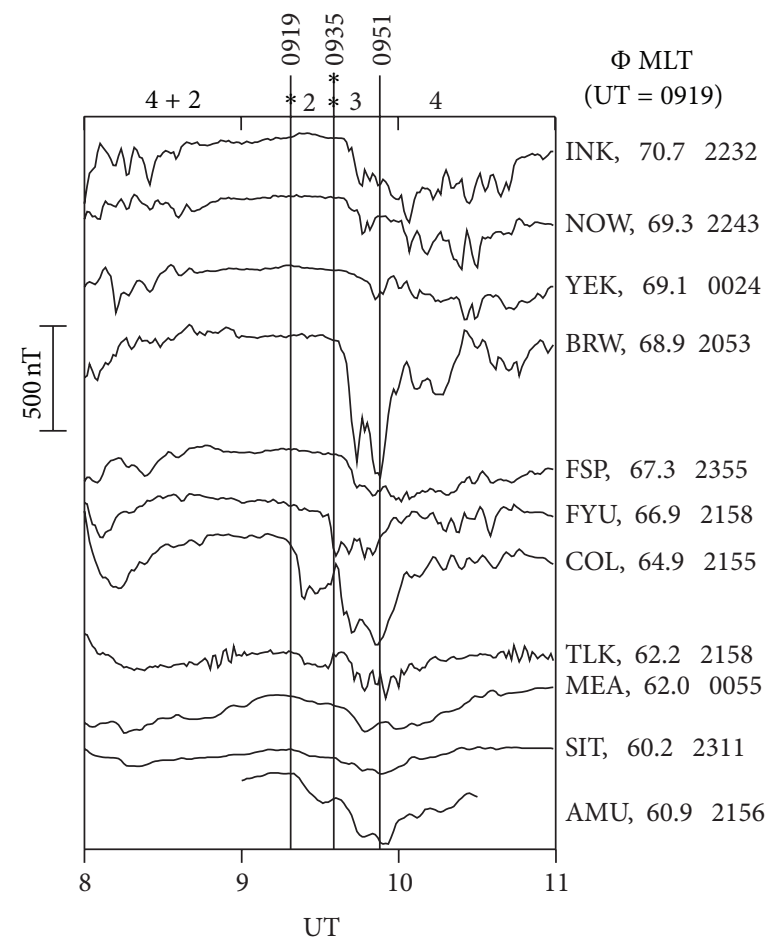

(c)

FIgURE 2: ((a), (b), and (c)) Timing of the chain of substorms CDAW9C. Magnetograms of the ground-based magnetometers for the chain of substorms; May 3, 1986. Single and double asterisks indicate the actively convective phase and the expansion (explosive) phase of the substorm, respectively [13]. 
TABLE 1

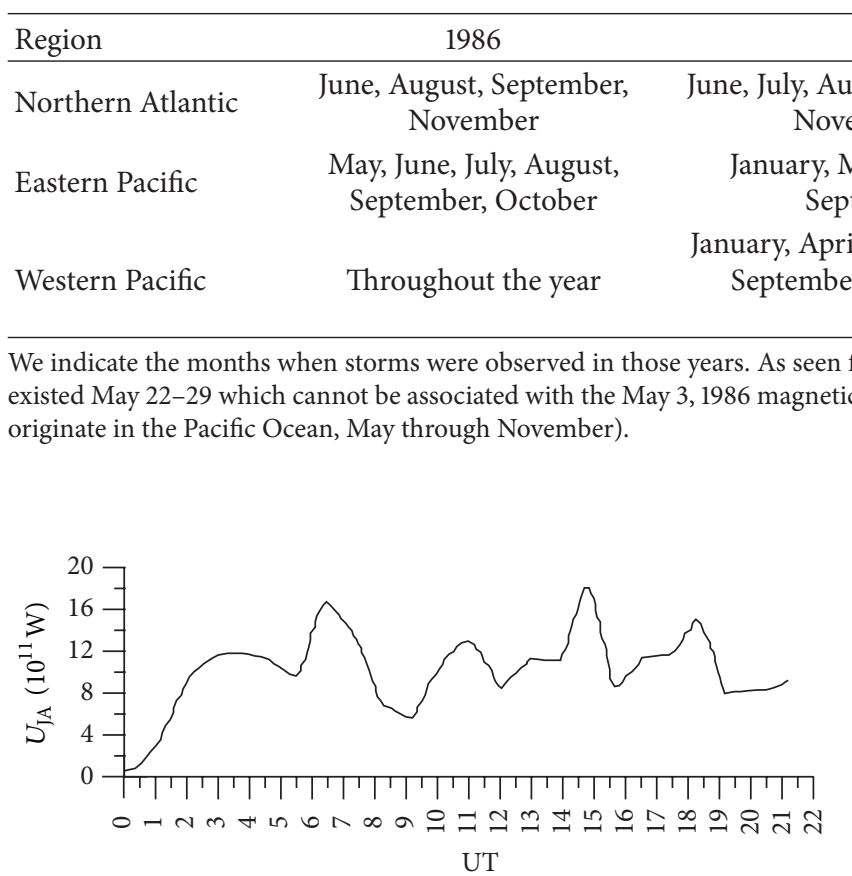

FIGURE 3: Estimations of power Joule heating and of power of particles precipitation into the ionosphere for the magnetospheric storm, March 13, 1989.

The 1989 March 13-14 magnetospheric storm refers to one of the strongest magnetospheric storms observed since the space era onset and occurred during solar Cycle-22, see, for example, $[16,17]$. The geomagnetic storm reached the maximal intensity on 1989 March 13 when the Dst-index reached a record value of about $-600 \mathrm{nT}[16,17]$. To save space, we do not give the illustrations for the 1989 March 13-14 event here. There are many papers describing this storm.

The 2003 October-November solar activity sharp burst led to strong magnetospheric storms with the 2003 October 28-31 high AE-index values and Dst-index of about -400 nT [18]. For the reasons above, we do not give the illustrations for this event here.

\section{Discussion and Conclusions}

As one can see (Figures 1, 3, and 4), the ionosphere underwent rather a significant Joule heating with a great power of precipitating energetic particles. Nevertheless, there were no abnormal variations in the lower atmosphere meteoparameters. The variations in physical characteristics of hurricanes (wind velocity, temperature, and pressure distribution) were not associated with high magnetic activity both in the highlatitude regions and in the mid-latitude ones (Figures 5, 6, and $7)$. We have used a lot of data for the analysis; some examples are illustrated in figures. There is no principal difference in plots for the periods of magnetospheric disturbances in comparison to nonstorm days. The atmospheric process power incomparably exceeds energy flux from the solar wind into the geomagnetosphere and the power of extremely strong magnetospheric disturbances. The energy flux from the magnetosphere into the atmosphere during the strong storm was about $1.5 \times 10^{19}(\mathrm{erg} / \mathrm{s}) \times 24 \times 3600=1.2 \times$ $10^{24}$ ergs/day, which is by 2-3 orders of magnitude less than the atmospheric process power whose values are in [19]. Investigations into the solar activity effect on atmospheric processes have become especially topical recently $[7,20]$. But, if there is a mechanism for the magnetospheric disturbance effect on meteorological processes in the atmosphere, it supposes a more complicated series of many intermediates and is not associated directly with the energy that arrives at the ionosphere during storms.

The main result of our investigation (see Table 1) is that the 1989 March magnetic storm did not directly affect the tropical cyclogenesis evolution character (Figures 8, 9, and 10). As for the 2003 October-November storms, one cannot answer unambiguously, either, because 5 plus intense and long-lived cyclones were observed then. The 13 October-1 November strong storm in the North Atlantic had existed extremely long (19 days), taking into account that the cyclone life average duration in Atlantic is 9 days. In 2003 October, 3 tropical cyclones were observed in the East Pacific: Hurricane Nora (October 1-9, the maximal wind being $41 \mathrm{~m} / \mathrm{s}$ ); Tropical Storm Olaf (October 3-8, $30 \mathrm{~m} / \mathrm{s}$ ); Tropical Storm Patricia (October $20-26,31 \mathrm{~m} / \mathrm{s}$ ). However, all of the strong storms in the East Pacific were observed prior to the magnetic storm onset.

Cloud layers play an important role in Earth's radiation balance [21-23], affecting the amount of heat from the Sun that reaches the surface and the heat radiated back from the surface that escapes out into space. One should pay special attention to the effect of the solar wind electric field sharp increase (via the global electric circuit during magnetospheric disturbances) on the cloud layer formation. It is necessary to test the assumptions that this layer may function as a screen decelerating radiative cooling of the air located on the Central Antarctic ice dome (as a result, there would be warming in the ground atmospheric layer and cooling above the cloud layer [24]). Authors in the paper [24] suggested that the interplanetary electric field influences the katabatic system of atmospheric circulation (typical of the winter in the Antarctic), via the global electric circuit affecting clouds and hence the radiation dynamics of the troposphere. 


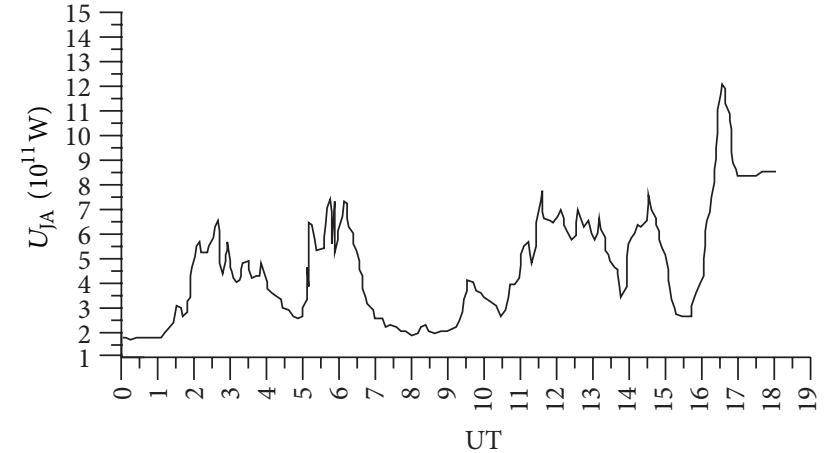

(a)

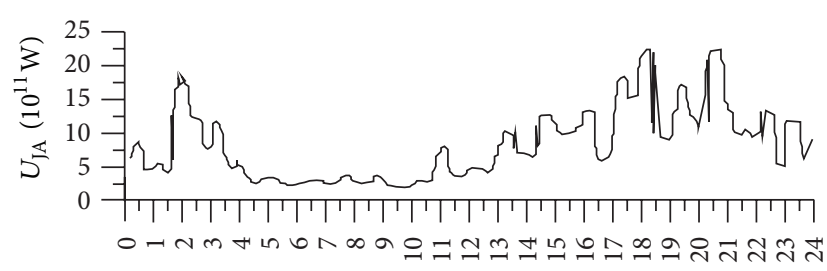

UT

(c)

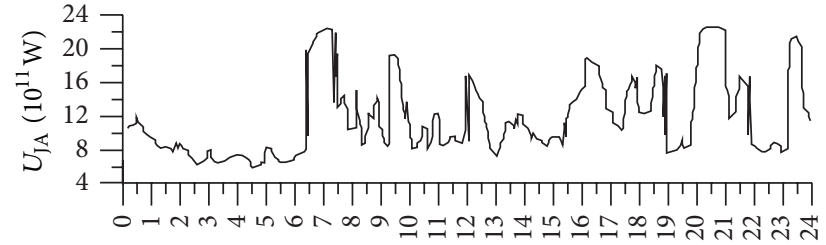

UT

(b)

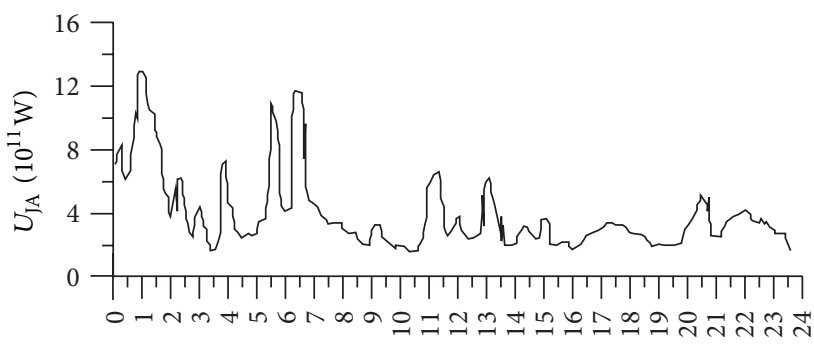

UT

(d)

Figure 4: Estimations of power of Joule heating and power of particles precipitation into the ionosphere: (a) October 28, 2003; (b) October 29, 2003; (c) October 30, 2003; (d) October 31, 2003.

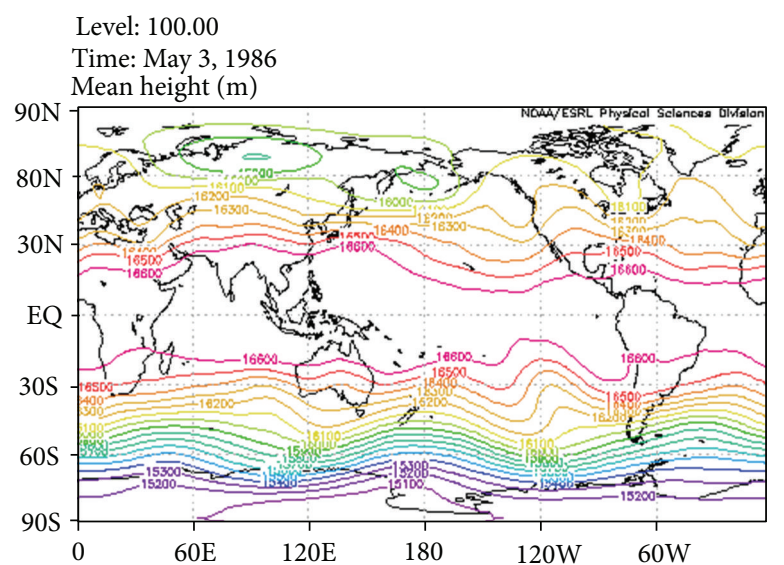

(a)

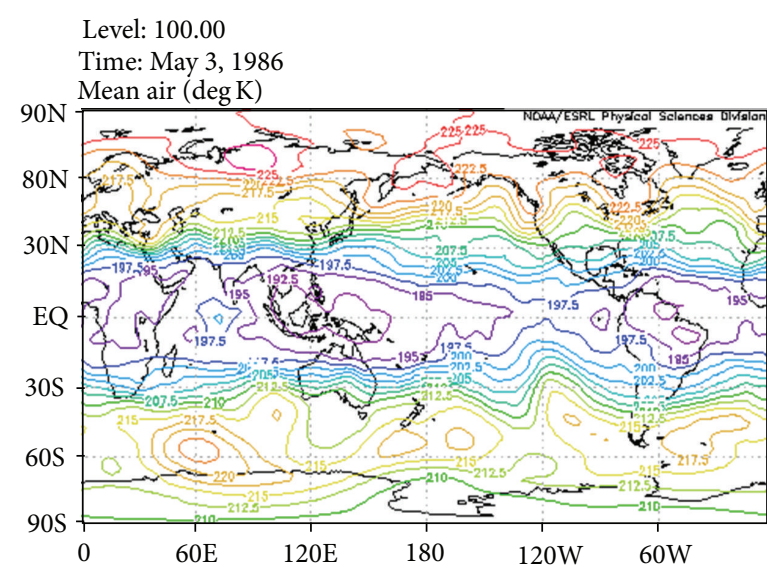

(b)

FIGURE 5: The NCEP/NCAR reanalysis example for the event, May 3, 1986: (a) height of an isobaric surface at the level $100 \mathrm{hPa}$ (average per day); (b) the average temperature at the level $100 \mathrm{hPa}$.

Let us briefly address the problem of the extraneous electric field penetration into the Earth's magnetosphere. More than five decades ago, Dungey [25] suggested the following model for the magnetospheric electric field generation. The solar wind magnetic field partially permeates inside the magnetosphere therefore, the polar cap field lines leave for the solar wind. In the solar wind, there is the electric field $E=-(1 / c)[V B]$, where $V$ is the solar wind velocity and $B$ is the interplanetary magnetic field. Because the conductance along field lines is very high, the electrical potential associated with the field in the solar wind is transported into the polar cap ionosphere. In the polar cap, a Sun-away convection originates, and, for that convection to be closed, reverse motions on closed field lines of the inner geomagnetosphere are necessary. Two-vortex convection was assumed to be obtained. Through multiplying the solar wind electric field value by the geomagnetosphere size towards dawn-dusk being about $25-30 \operatorname{Re}(\operatorname{Re} \sim 6371 \mathrm{~km})$ and through assuming the solar wind velocity as $\sim 450 \mathrm{~km} / \mathrm{s}$, we will obtain the potential difference between the dawn and the dusk sides of 


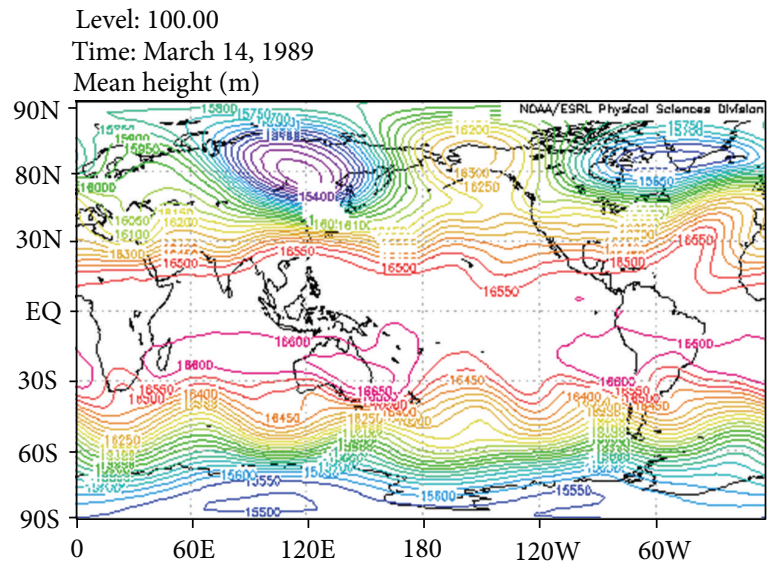

(a)

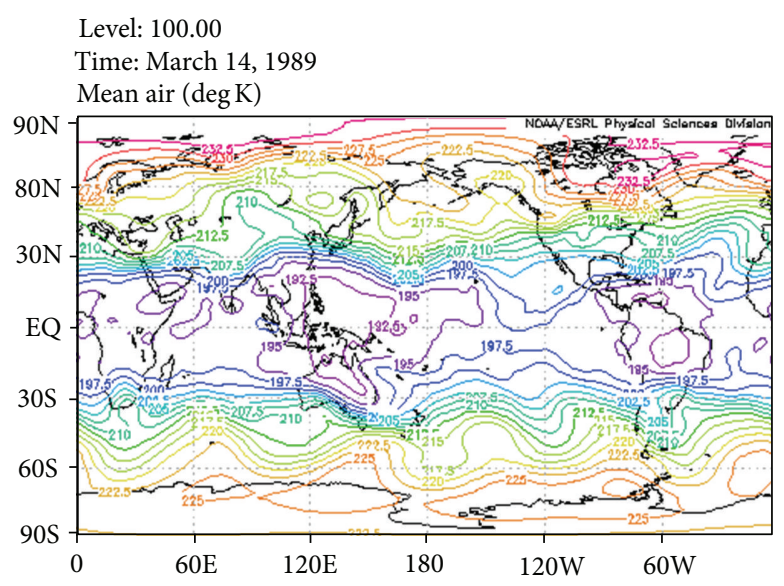

(b)

FIGURE 6: The NCEP/NCAR reanalysis example for the event, March 13-14, 1989: (a) height of an isobaric surface at the level $100 \mathrm{hPa}$ (average per day); (b) the average temperature at the level $100 \mathrm{hPa}$.

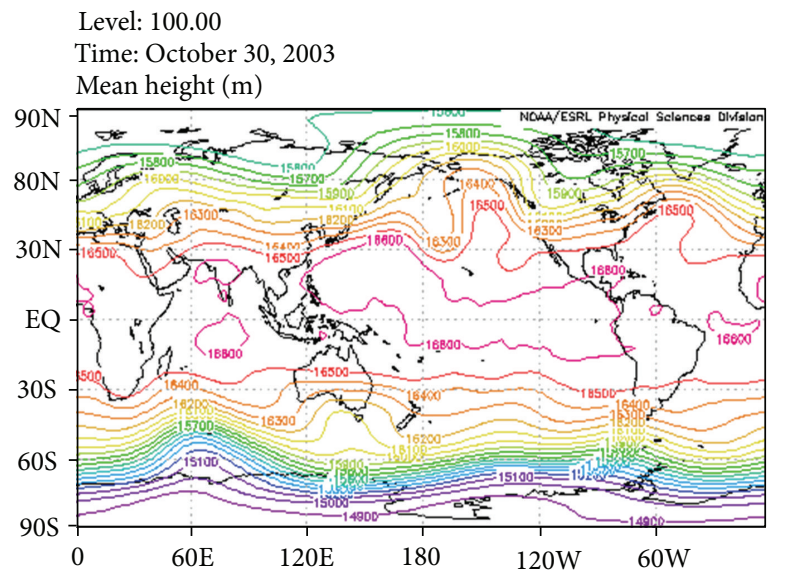

(a)
Level: 100.00

Time: October 30, 2003

Mean air $(\operatorname{deg} K)$

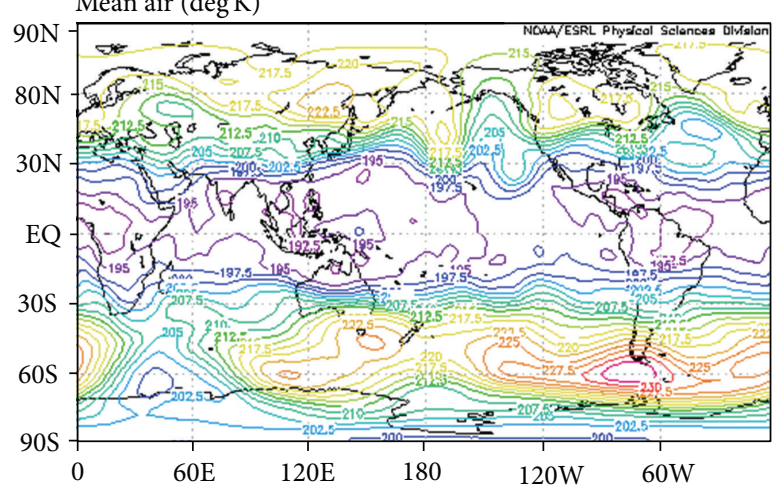

(b)

FIGURE 7: The NCEP/NCAR reanalysis example for the event, October 30, 2003: (a) height of an isobaric surface at the level $100 \mathrm{hPa}$ (average per day); (b) the average temperature at the level $100 \mathrm{hPa}$.

the magnetosphere $\Delta \Psi \approx-80 \mathrm{Bz},(\mathrm{kV})$ which is manifold less than the well-known experimental formula [26] $\Delta \Psi(\mathrm{kV})=$ $-11 \mathrm{Bz}(\mathrm{nT})$ provides.

The penetration of the electric field and the current into the geomagnetosphere is a two-stage process and may be presented as follows (see Figure 11). Let an electric current component towards the magnetosphere appear at instant $\mathrm{T}$. A potential value will be established at the magnetopause segment. In the thin near-side layer of the thickness $d \sim$ $2 \pi c / \omega_{\mathrm{pp}}$ (where $\omega_{\mathrm{pp}}$ is proton plasma frequency and $c$ is the speed of light), the charge division process will start, and the displacement current $j^{*}=(\varepsilon / 4 \pi) \times \partial E / \partial t$ will appear. Also, there will appear Ampere force $F=\left[j^{*} \times B\right] / c$ that will start accelerating plasma. The only force that withstands the Ampere one is the inertia force. Under the conditions of a homogeneous medium, the inertia force is $\rho \partial v / \partial t$ :

$$
\frac{\rho \partial v}{\partial t}=\frac{\left[j^{*} \times B\right]}{c}=\left(\frac{\varepsilon}{4 \pi c}\right) \times\left[\frac{\partial E}{\partial t} \times B\right] .
$$

Taking into account that $\varepsilon=c^{2} / V_{A}^{2}$, where $V_{A}$ is the Alfven velocity, upon integrating we will have $v=c[E \times B] / B^{2}$ that is, the classic equation for the electric drift velocity (it is important for us to express the dynamic process in this case).

When the plasma is accelerated in the layer $d$ up to the $V \times B$ drift velocity (and it happens during the gyroperiod), then there will be no field in the plasma coordinate system, and it appears at the boundary between the moving and stable plasmas in a stable coordinate system (see Figure 11). The boundary moving velocity separating the moving plasma from the stable one will be, consequently, $V_{\phi} \sim d \omega_{B} / 2 \pi$, where $\omega_{B}$ is the proton gyrofrequency. Taking the values $d$ and $\omega_{B}$ into the equation for the phase velocity, we see that it is the Alfven velocity like we expected. Thus, the external electric field penetrates into the magnetosphere without any limitations of the Alfven-wave type, and the electric current is only in a form of the displacement current. The electric current flows through the system under consideration only when there is a transitive process. In the stationary regime, there is 


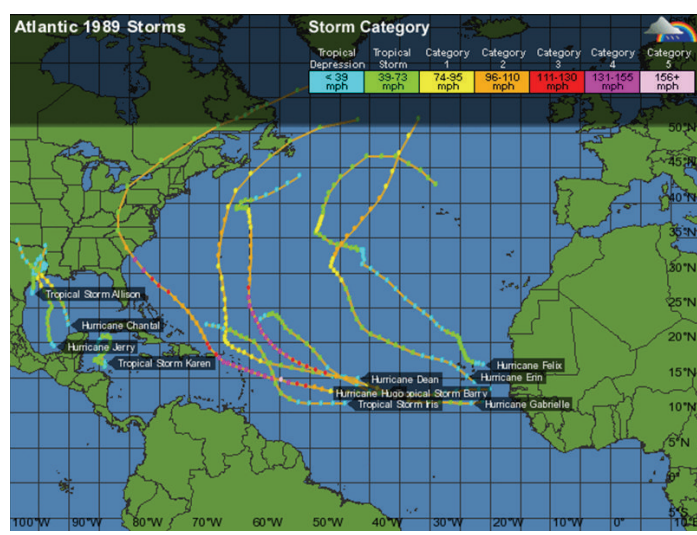

(a)

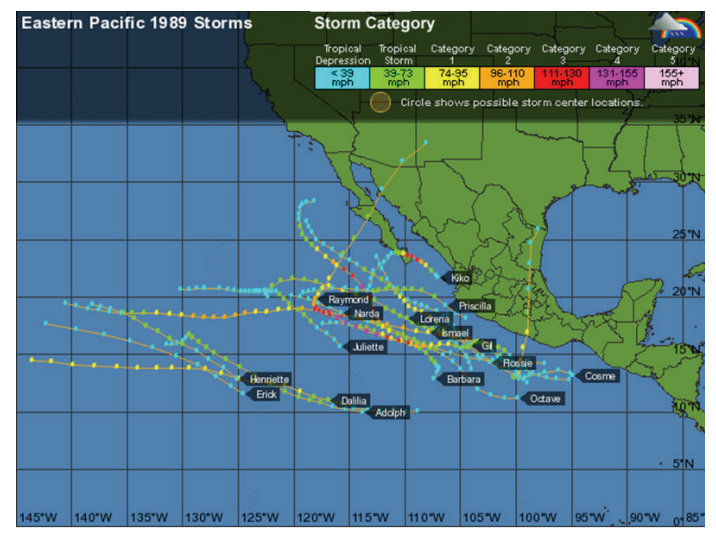

(b)

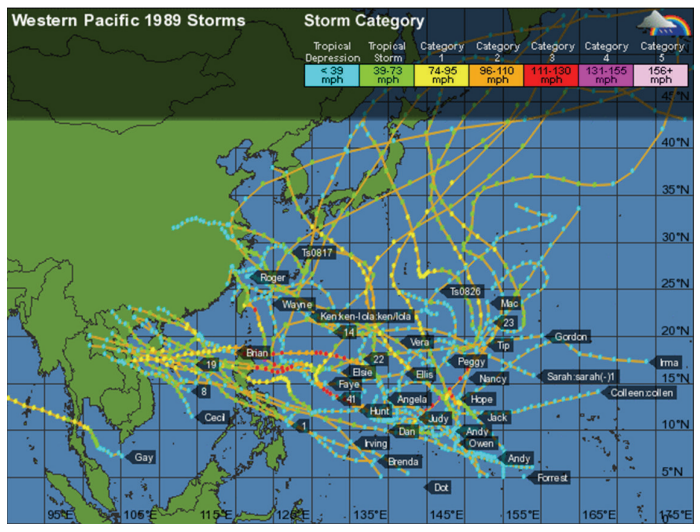

(c)

FIgURE 8: (a) The example of the trajectory of the cyclone observed in the North Atlantic, for events in 1989. (b) The example of the trajectory of the cyclone observed in Eastern Pacific, for events in 1989. (c) The example of the trajectory of the cyclone observed in Western Pacific, for events in 1989.

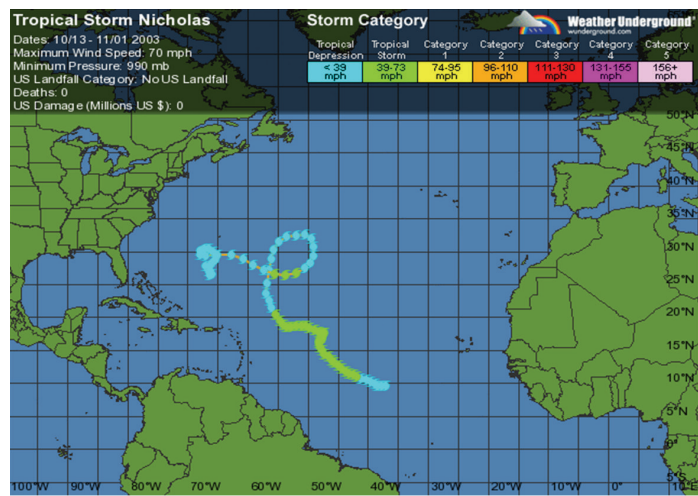

FIGURE 9: The trajectory of the cyclone observed in the North Atlantic 13 October-1 November, 2003. This storm was observed during the magnetic storm and has existed maximum for a long time (19 days) at average life time of a cyclone, which is 9 days, in the North Atlantic.

no electric current. If the magnetic field is inhomogeneous according to an axis $X$, then the gas pressure gradient will be originated independently due to the flow nonuniformity. The electric field establishment time in the system here is
$\tau_{E}=L / V_{\phi}$, and the current establishment time is $\tau_{I}=L^{*} / V_{c}$, where $L$ is the system size, $V_{\phi}$ is the phase velocity for the electromagnetic signal propagation across the system, $L^{*}=$ $(B / \nabla B)$, and $V_{c}$ is the plasma convection velocity. An approximate estimate applied to the magnetosphere gives the time of the electric field establishment to be hundreds of seconds and the electric current establishment time to be about an hour. Thus, the electric current penetration into plasma is a twostage process. Initially, the polarization field that penetrates into plasma "layer by layer" is produced. Or, to be more exact, the momentum corresponding to this field penetrates into plasma. Here, if the system is inhomogeneous, the flow can redistribute pressure so that an electric current arises in plasma because of the appearance of gradients. This electric current is necessary to maintain plasma convection in the magnetosphere [27, 28].

Indeed, there is no simple global electric circuit via which a sharp increase in the solar wind electric field during magnetospheric disturbances would be possible. The solar wind electric field penetration process is complex and nonlinear. Field-aligned currents connect the magnetosphere and the ionosphere in the united electric circuit. The plasma convection generation in the geomagnetosphere is associated with the processes at the bow shock front [27]. The combined 


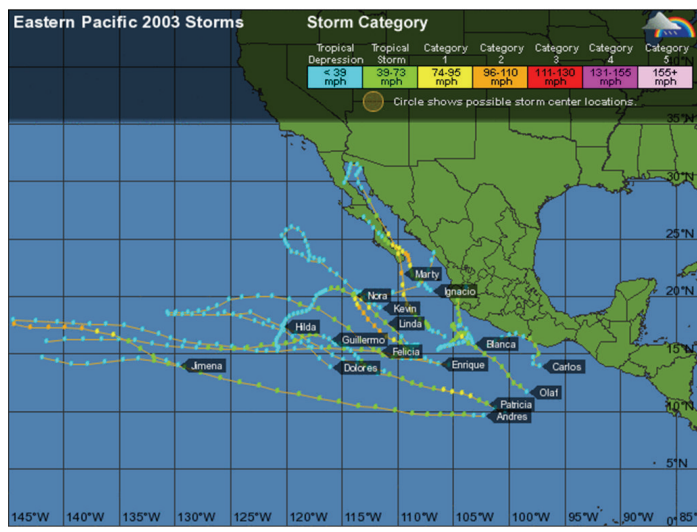

(a)

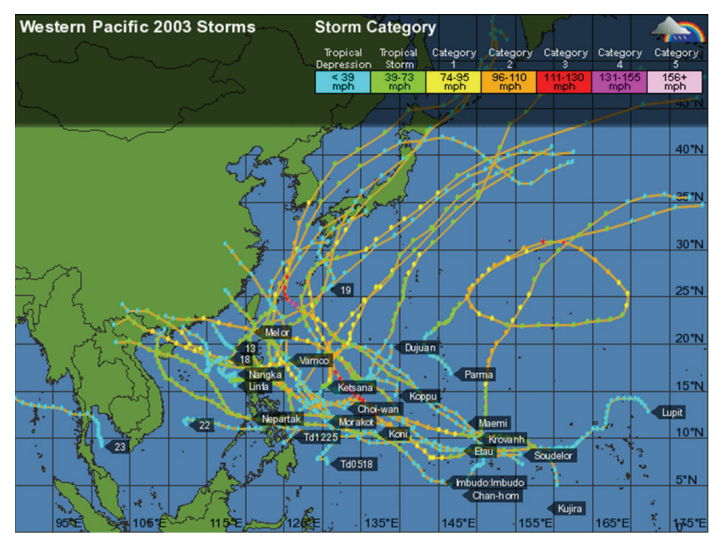

(b)

Figure 10: (a) The example of trajectories of the cyclone observed in Eastern Pacific. In October, 2003, in East part of Pacific ocean 3 outputs of a tropical cyclone were observed: Hurricane Nora, October, 1-9, 2003, with the maximal speed of wind $-41 \mathrm{~m} / \mathrm{s}$; Tropical Storm Olaf, October, 3-8, 2003, with maximal wind speed of $30 \mathrm{~m} / \mathrm{s}$; Tropical Storm Patricia, October, 20-26, 2003, with the maximal wind speed of $31 \mathrm{~m} / \mathrm{s}$. All storms were observed prior to the beginning of action of the magnetospheric storm. (b) The example of trajectories of the cyclone observed in Western Pacific for events in 2003. In October-November, 2003, more than 5 intensive and long cyclones were observed.

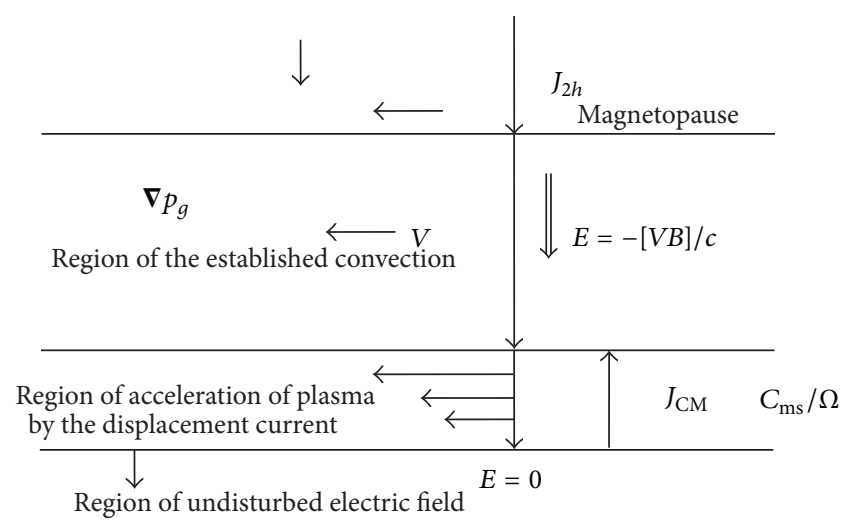

FIGURE 11: The scheme of the penetration of the external electric current and electric field in the magnetospheric plasma and generation of magnetospheric convection. In the figure, the part of magnetopause, through which the electric current and the electric field will penetrate, is shown.

action of plasma convection and pitch-angle diffusion of electrons and protons leads to the formation of plasma pressure distribution in the magnetosphere. As known, steady electric bulk currents are associated with the gas pressure distribution. The divergence of these bulk currents causes a spatial distribution of field-aligned currents, that is, magnetospheric sources of the ionospheric electric current systems [29]. The atmospheric conductivity sharply declines between the polar ionosphere and the layer at $\mathrm{h} \sim 10 \mathrm{~km}$.

The geomagnetospheric disturbance effect on the troposphere is weak compared with a multitude of other factors affecting it. However, the existing works on a high correlation between tropical cyclones and magnetic storms may evidence either the existence of another mechanism for the effect (that was not addressed in this study) or a random coincidence rather than a physical essence. A very interesting mechanism suggested by Troshichev and Janzhura [24] needs further considering and improving. Authors in the paper [24] noted that the solar wind dynamic pressure effect on the cloud layer would be opposite to that of the interplanetary electric field. Thus, now we can note that, probably, there is some connection between processes at the bow shock front region and meteorological processes at the lower atmosphere, because the magnetospheric plasma convection generation is associated with processes at the bow shock front (e.g., see [27]).

A further study of geomagnetospheric storm effects on tropical cyclogenesis is necessary, because the character of some influence has regional peculiarities (see Figures 8-10). In the next paper, we are going to investigate a probable effect of such magnetospheric disturbances as sawtooth events, extraordinary powerful magnetic storms, and some others on meteorological processes in the lower atmosphere.

\section{Acknowledgments}

This study was supported by the Russian Foundation for Basic Research, Project no. 12-05-31096 mol_a. The authors are grateful to creators and developers of the following websites: (1) http://www.nhc.noaa.gov/pastall.shtml; (2) http://www .csc.noaa.gov/hurricane_tracks; (3) http://www.aoml.noaa .gov/hrd/hurdat/ushurrlist18512007.txt; (4) http://russian .wunderground.com/hurricane/hurrarchive.asp? region=at; (5) ftp://ftp.cdc.noaa.gov/; (6) http://www.kosmofizika.ru/; (7) http://www.viems.ru/asnti/ntb/ntb502/oboc5.html for providing observational data.

\section{References}

[1] V. Bucha, "Geomagnetic activity and the global temperature," Studia Geophysica et Geodaetica, vol. 53, no. 4, pp. 571-573, 2009. 
[2] V. Bucha, "Changes in geomagnetic activity and global temperature during the past 40 years," Studia Geophysica et Geodaetica, vol. 56, no. 4, pp. 1095-1107, 2012.

[3] D. R. Palamara, "An interhemispheric comparison of the geomagnetic activity signature in the lower atmosphere," Earth, Planets and Space, vol. 56, no. 9, pp. e25-e28, 2004.

[4] J. D. Haigh, M. Blackburn, and R. Day, "The response of tropospheric circulation to perturbations in lower-stratospheric temperature," Journal of Climate, vol. 18, no. 17, pp. 3672-3685, 2005.

[5] E. A. Sharkov, "Global tropical cyclogenesis: evolution of scientific views," Issledovanie Zemli iz Kosmosa, no. 6, pp. 1-9, 2005 (Russian).

[6] K. G. Ivanov, "Correlation between tropical cyclones and magnetic storms during cycle 23 of solar activity," Geomagnetism and Aeronomy, vol. 47, no. 3, pp. 371-374, 2007.

[7] K. G. Ivanov, "Generation of the Katrine hurricane during the geomagnetic extrastrom at crossing of the heliospheric current sheet: is it an accidental coincidence or physical essence?" Geomagnetism and Aeronomy, vol. 46, no. 5, pp. 609-615, 2006.

[8] M. I. Pudovkin, "Solar activity effect on the condition of the lower atmosphere and weather," Soros's Educational Journal, no. 10, pp. 106-113, 1996.

[9] D. Hauglustaine and J. C. Gerard, "Possible composition and climatic changes due to past intense energetic particle precipitation," Annales Geophysicae, vol. 8, no. 2, pp. 87-96, 1990.

[10] A. D. Richmond, G. Lu, B. A. Emery, and D. J. Knipp, “The Amie procedure: prospects for space weather specification and prediction," Advances in Space Research, vol. 22, no. 1, pp. 103112, 1998.

[11] B. H. Ahn, H. W. Kroehl, Y. Kamide, and D. J. Gorney, "Estimation of ionospheric electrodynamics parameters using ionospheric conductance deduced from Bremsstrahlung X-ray image data," Journal of Geophysical Research: Space Physics, vol. 94, no. A3, pp. 2565-2586, 1989.

[12] R. W. Spiro, P. H. Reiff, and L. J. Maber, "Precipitating electron energy flux," Journal of Geophysical Research: Space Physics, vol. 87, no. A10, pp. 8215-8227, 1982.

[13] P. A. Sedykh and L. V. Minenko, "Studying CDAW9C substorms," in Proceedings of the BSFP, vol. 2, pp. 562-576, Irkutsk, Russia, 1999.

[14] Yu. P. Maltsev, L. L. Lazutin, V. G. Vorobyev et al., Physics of the Near-Earth Space, vol. 1, RAS KRC, PSP, 2000.

[15] P. A. Sedykh, "On two-stage evolution of the substorm active phase," in Proceedings of the BSFP, pp. 90-94, Irkutsk, Russia, 2009.

[16] Yu. P. Tsvetkov, A. N. Zaitsev, V. I. Odintsov, C. K. Hao, and N. T. K. Thoa, "Comparison of magnetic variations in the equatorial zone and the polar cap for the magnetic storm of March 13, 1989," Geomagnetism and Aeronomy, vol. 38, no. 2, pp. 192-200, 1998.

[17] L. A. Hajkowicz, "Global onset and propagation of large-scale travelling ionospheric disturbances as a result of the great storm of 13 March 1989," Planetary and Space Science, vol. 39, no. 4, pp. 583-593, 1991.

[18] M. I. Panasyuk, C. N. Kuznetsov, L. L. Lazutin et al., "Magnetic storms in October 2003: Collaboration 'Solar Extreme Events in 2003 (SEE-2003)', Space Research, vol. 42, no. 5, pp. 489-534, 2004.

[19] M. I. Pudovkin and S. V. Babushkina, "Influence of solar flares and disturbances of the interplanetary medium on the atmospheric circulation," Journal of Atmospheric and Solar-Terrestrial Physics, vol. 54, no. 7-8, pp. 841-846, 1992.
[20] M. V. Vorotkov and V. L. Gorshkov, "Solar flare activity effect on cyclone activity of the Earth's atmosphere," in Proceedings of the Problems of Astronomy, pp. 231-239, Irkutsk, Russia, 2011.

[21] B. A. Tinsley, G. M. Brown, and P. H. Scherrer, "Solar variability influences on weather and climate: possible connections through cosmic ray fluxes and storm intensification," Journal of Geophysical Research, vol. 94, no. 12, pp. 14-792, 1989.

[22] B. A. Tinsley and G. W. Deen, "Apparent tropospheric response to $\mathrm{MeV}-\mathrm{GeV}$ particle flux variations: a connection via electrofreezing of supercooled water in high-level clouds?" Journal of Geophysical Research, vol. 96, no. 12, pp. 22283-22296, 1991.

[23] B. A. Tinsley and R. A. Heelis, "Correlations of atmospheric dynamics with solar activity evidence for a connection via the solar wind, atmospheric electricity, and cloud microphysics," Journal of Geophysical Research, vol. 98, no. 6, pp. 10375-10384, 1993.

[24] O. A. Troshichev and A. Janzhura, "Temperature alterations on the Antarctic ice sheet initiated by the disturbed solar wind," Journal of Atmospheric and Solar-Terrestrial Physics, vol. 66, no. 13-14, pp. 1159-1172, 2004.

[25] J. W. Dungey, "Interplanetary magnetic field and the auroral zones," Physical Review Letters, vol. 6, no. 2, pp. 47-48, 1961.

[26] M. A. Doyle and W. J. Burke, "S3-2 measurements of the polar cap potential," Journal of Geophysical Research, vol. 88, no. 11, pp. 9125-9133, 1983.

[27] E. A. Ponomarev, P. A. Sedykh, and V. D. Urbanovich, "Generation of electric field in the Earth magnetosphere, caused by processes in the bow shock," Journal of Atmospheric and SolarTerrestrial Physics, vol. 68, no. 6, pp. 679-684, 2006.

[28] P. A. Sedykh, "On the role of the bow shock in power of magnetospheric disturbances," Sun \& Geosphere, vol. 6, no. 1, pp. 27-31, 2011.

[29] P. A. Sedykh and E. A. Ponomarev, "A structurally adequate model of the geomagnetosphere," Studia Geophysica et Geodaetica, vol. 56, no. 4, pp. 110-126, 2012. 

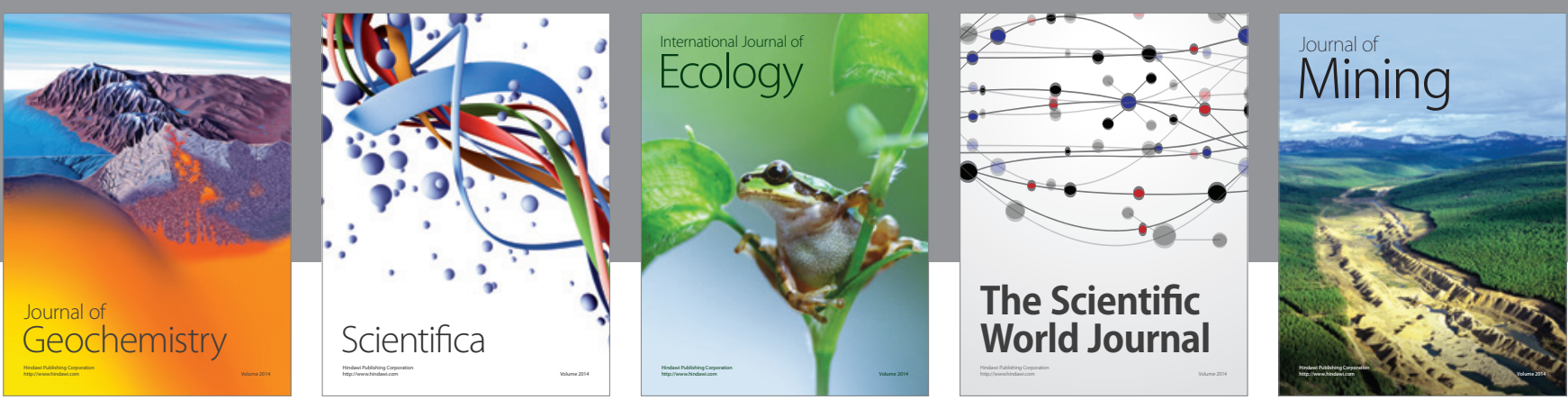

The Scientific World Journal
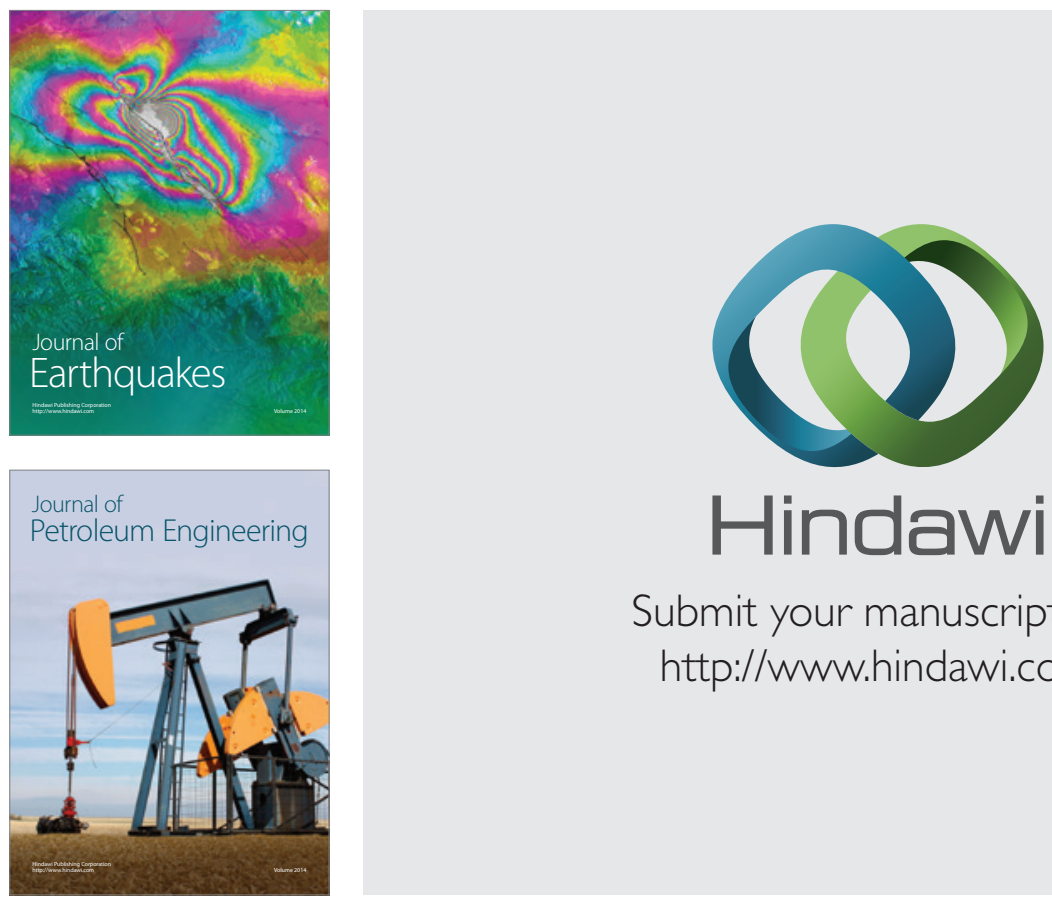

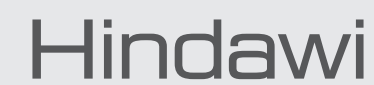

Submit your manuscripts at

http://www.hindawi.com
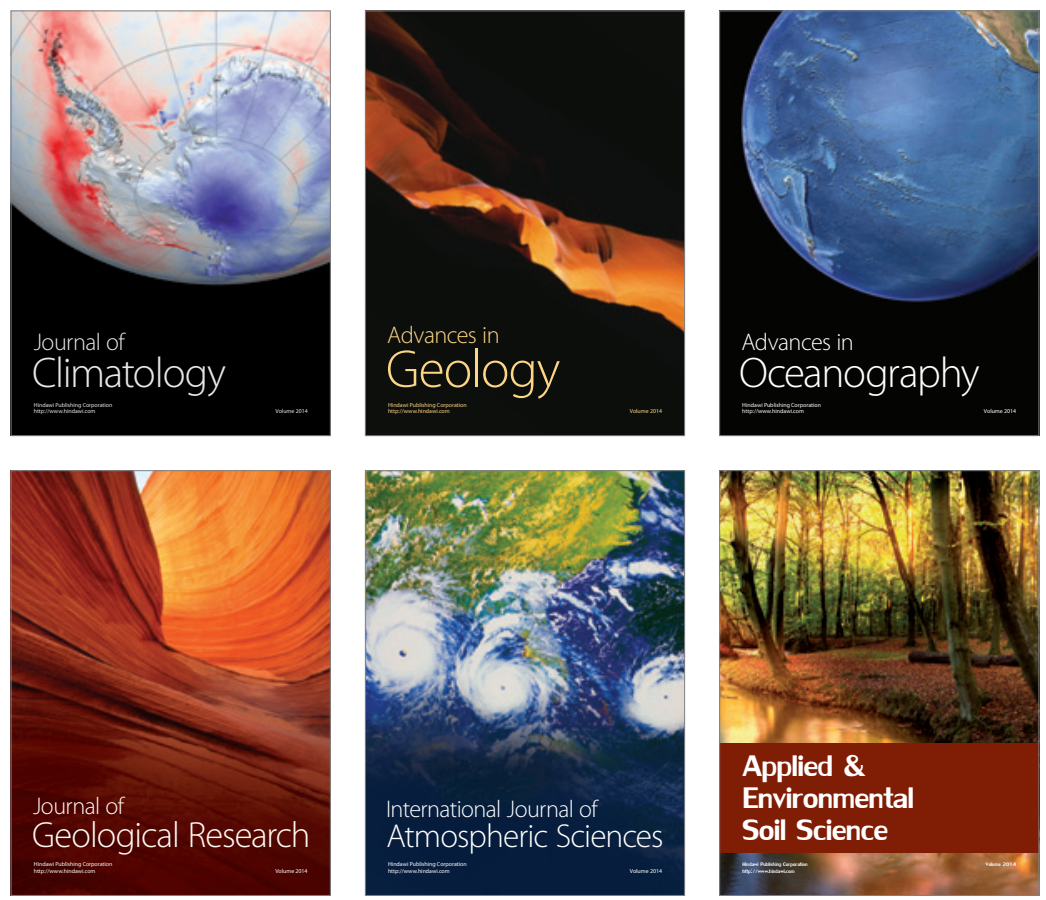
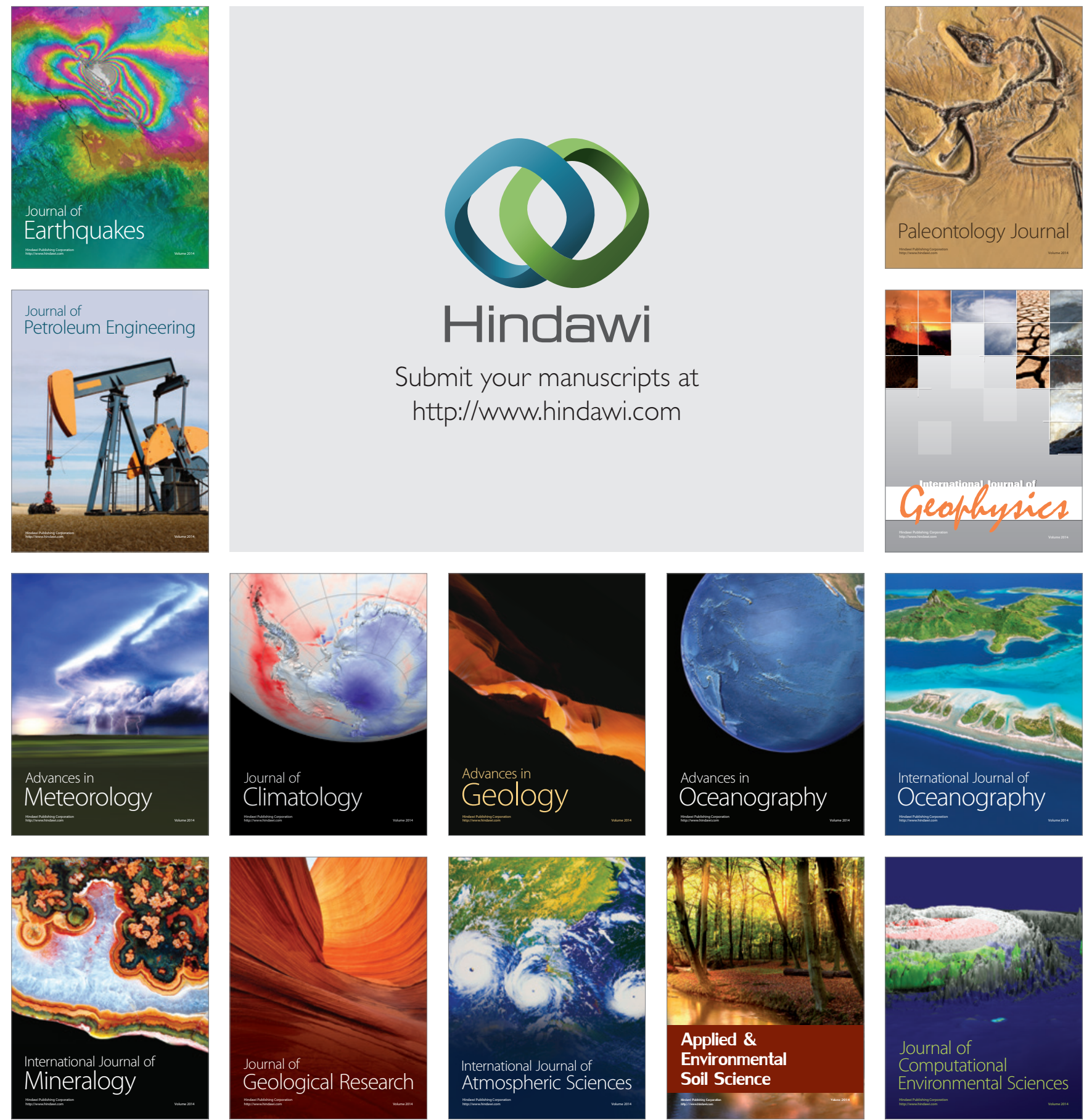